\title{
Giant hydronephrosis in adults: the great mimic. Early diagnosis with ultrasound
}

\author{
WT Yang, C Metreweli
}

\begin{abstract}
Summary
Giant hydronephrosis in adults is uncommon and often misdiagnosed clinically. We present three cases of giant hydronephrosis in adults secondary to ureteric calculous obstruction, two of whom presented with an acute abdomen and one with an indirect inguinal hernia. All patients were diagnosed promptly with ultrasound.
\end{abstract}

Keywords: giant hydronephrosis, ultrasound

Most reported cases of giant hydronephrosis occur in infants and children, and are congenital in origin. Giant hydronephrosis in adults secondary to calculous obstruction is uncommon and often misdiagnosed clinically. Patients may present with asymptomatic abdominal distension of long standing, an asymptomatic abdominal mass or an acute abdomen. Plain abdominal radiographs may not always provide an accurate diagnosis due to obscuration of radio-opaque urinary calculi by bowel shadows, or overlap with the spine. Ultrasound in such patients is a quick, noninvasive and sensitive method for confirming the diagnosis in order that the appropriate management may be instituted promptly.

\section{Case 1}

A 58-year-old hypertensive Chinese woman on anti-hypertensive medication complained of intermittent abdominal pain and abdominal swelling for six days, with no precipitating or relieving factors. The pain was associated with constipation and a decrease in appetite. She subsequently developed fever and dysuria. Examination revealed fever, a distended abdomen with no palpable masses, and no shifting dullness. Laboratory investigations were normal. The clinical impression was possible biliary sepsis. Plain abdominal radiograph revealed a $1-\mathrm{cm}$ rounded radio-opacity superimposed over the right L4 transverse process (figure 1A). Ultrasound revealed a grossly distended right pelvicalyceal system containing echogenic material with no recognisable renal parenchyma. Multiple obstructing calculi were seen at the right pelvi-ureteric junction (PUJ) (figure 1B) with multiple calculi also noted in the lower pole calyx. A right percutaneous nephrostomy yielded a total of 141 of turbid urine and showed complete hold-up of contrast by the right PUJ calculus (figure 1C). The differential renal function of the right kidney was $12 \%$ and left kidney $88 \%$ by technetium- $99 \mathrm{~m}$ dimercapto-succinate (DMSA) scan.

The patient subsequently underwent a right nephrectomy. Histology confirmed hydronephrosis, chronic pyelonephritis with several small renal stones present in the lower pole calyx. The patient recovered well postoperatively and is currently asymptomatic.

\section{Case 2}

A 73-year-old Chinese woman complained of sudden onset of dull right upper quadrant pain for two days associated with nausea, vomiting and fever, but no urinary symptoms. Physical examination showed that the patient was febrile, with a distended abdomen and tenderness in the right upper quadrant. There were no palpable abdominal masses. The clinical impression was acute cholecystitis. The white cell count was raised to $17.5 \times 10^{9} / 1$. Urinalysis showed a large number of white and red cells, with no bacterial growth from urine culture.

Plain abdominal radiograph showed a left staghorn calculus but failed to demonstrate any right urinary calculi due to bowel obscuration (figure 2A). Ultrasound of the abdomen revealed a massively dilated right pelvicalyceal system (30 cm in length) and proximal ureter with echogenic debris in the collecting system. A $1.5 \mathrm{~cm}$ proximal right ureteric calculus was seen as the cause of obstruction (figure 2B). Incidental finding of gallstones and a left staghorn calculus was also noted. A right percutaneous nephrostomy demonstrated a massively hydronephrotic right collecting system with complete obstruction by the ureteric calculus. A total of 1.61 of urine was drained, and the patient subsequently recovered from her clinical symptoms. DMSA scintigram showed a differential function of $91 \%$ for the left kidney and $9 \%$ for the right kidney as well as diffuse parenchymal loss on the right and patchy focal defects on the left.

The patient is currently being managed conservatively with a view to possible nephrectomy should symptoms arise. Extracorporeal shock wave lithotripsy and a double J stent insertion for the left staghorn calculus have been scheduled.

\section{Case 3}

A 62-year-old Chinese man complained of a reducible right groin lump which was intermit- 


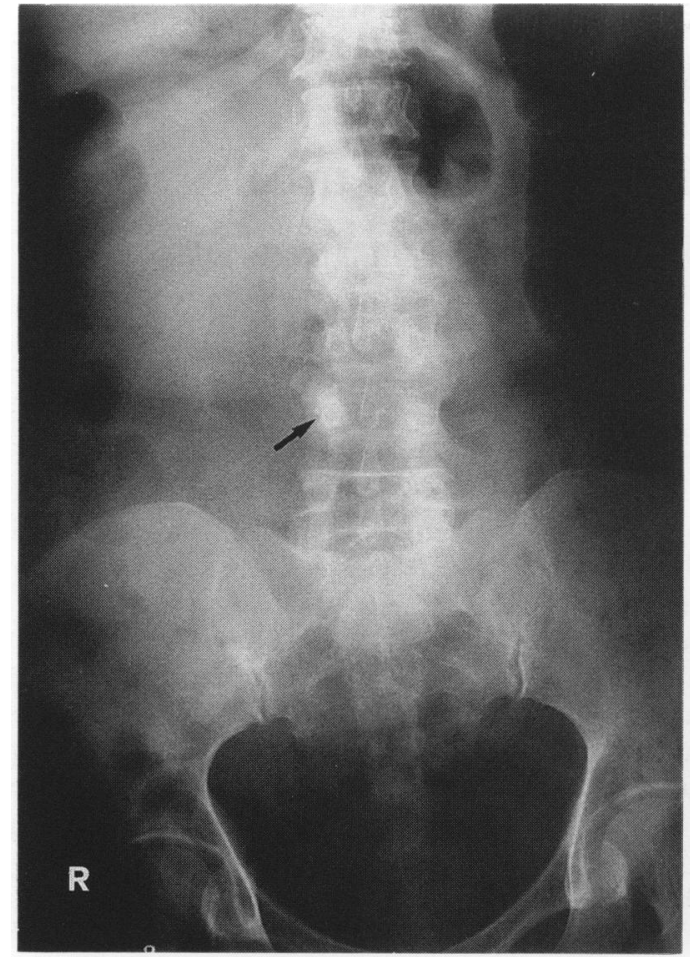

A

Figure 1 (A) Plain abdominal radiograph shows a small rounded opacity superimposed over the right L4 transverse process (arrow). (B) Longitudinal ultrasound scan through the right kidney shows a dilated pelvicalyceal system containing echogenic material, and the obstructing PUJ calculus (long arrow). (C) Right percutaneous nephrostomy shows a massively dilated collecting system due to complete obstruction by the right PUJ calculus (short arrow)

tently painful and had been increasing in size over six months. He denied any loin pain, haematuria or abdominal distension. Physical examination revealed a reducible right inguinal mass with a cough impulse. The patient was obese and no abdominal masses were palpable. The clinical diagnosis was a right indirect inguinal hernia. Laboratory investigations were normal. Plain abdominal radiograph showed a large soft tissue opacity filling the left hemi-abdomen, with an ovoid radio-opacity projected over the right L4 transverse process, and smaller rounded radio-opacities in the left lower quadrant (figure 3A).

Ultrasound of the abdomen showed gross left hydronephrosis (more than $30 \mathrm{~cm}$ ) extending from the left flank to the right iliac fossa. A $2.5 \mathrm{~cm}$ calculus was demonstrated in the proximal left ureter (figure 3B), corresponding to the radio-opacity seen over the right L4 transverse process. Multiple small calculi were seen in the lower pole calyx with no residual left renal parenchyma seen (figure 3C). A DMSA scan showed differential renal function of $100 \%$ on the right.

A left nephrectomy was performed in view of the left giant hydronephrosis causing increased

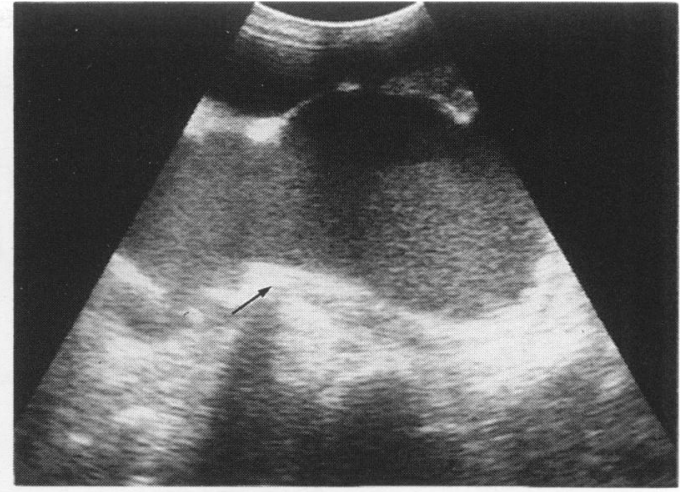

B

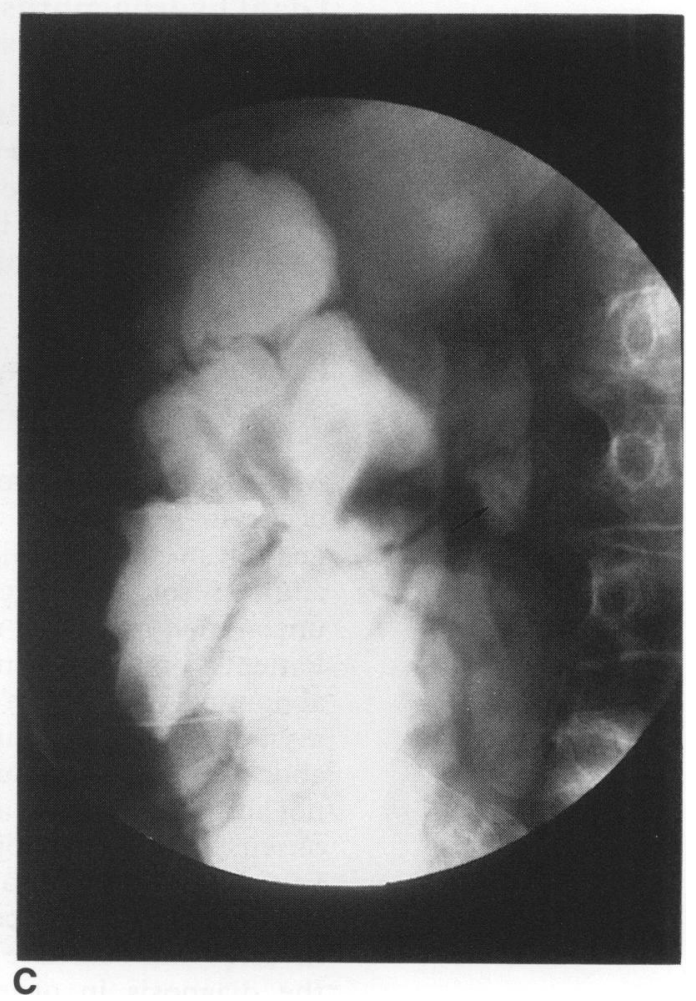

intra-abdominal pressure and a subsequent right inguinal hernia. The patient had an uneventful postoperative recovery.

\section{Discussion}

Giant hydronephrosis has been defined as a kidney containing more than $1000 \mathrm{ml}$ of fluid in its collecting system. ${ }^{1}$ The radiological diagnostic criteria are a hydronephrotic renal pelvis that meets or crosses the midline, occupies a hemi-abdomen and extends for a length of five vertebrae or more. ${ }^{2}$ Since the first description of giant hydronephrosis in $1746,{ }^{3}$ more than 180 cases have been described. ${ }^{4}$ With higher standards of medical care giant hydronephrosis is now a rare urological entity, occurring predominantly in children. The commonest cause is congenital PUJ obstruction which occurs in $80 \%$ of cases. ${ }^{1,5}$ Other causes include flap-like mucosal folds, polar or aberrant vessels, ureteric kinks and high pelvic insertions of the ureter. Giant hydronephrosis has been reported in the literature, ${ }^{6-9}$ but little has appeared regarding its occurrence in adults secondary to calculous obstruction.

Giant hydronephrosis is a slowly progressive 


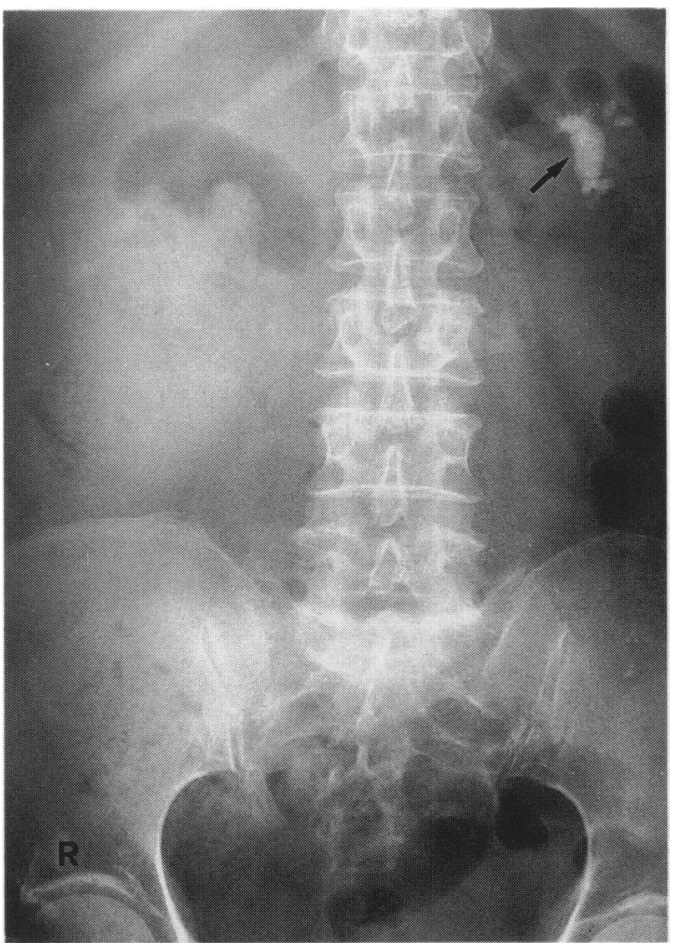

A

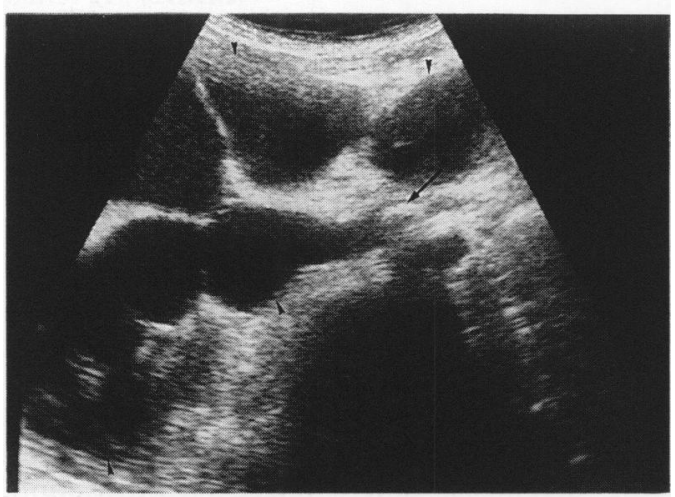

B

Figure 2 (A) Plain abdominal radiograph shows a left staghorn calculus (arrow) but no visible right urinary calculi. (B) Longitudinal ultrasound scan of the right kidney demonstrates a dilated pelvicalyceal system (arrowheads) containing echogenic debris, and an obstructing right PUJ calculus (long arrow)

disease, and a huge abdominal mass or distended abdomen may be the only sign. The clinical features include an asymptomatic enlargement of the abdomen of long-standing, which may be preceded by a mass in either flank. The patient may present after minimal trauma with flank pain, haematuria and sometimes shock. It does not always progress, but may increase in size rapidly and without warning in adults. When this occurs, an underlying renal or pelvic carcinoma with haemorrhage into the obstructed renal pelvis should be considered. ${ }^{2}$ Infrequently, it may present with respiratory distress or intraperitoneal urinary extravasation, particularly in infants with primary obstructive mega-ureter. ${ }^{10}$ Gross bilateral disease may cause uraemia. Giant hydronephrosis may fill the entire abdomen and differentiation of the condition from ascites may be clinically difficult. Erroneous diagnosis

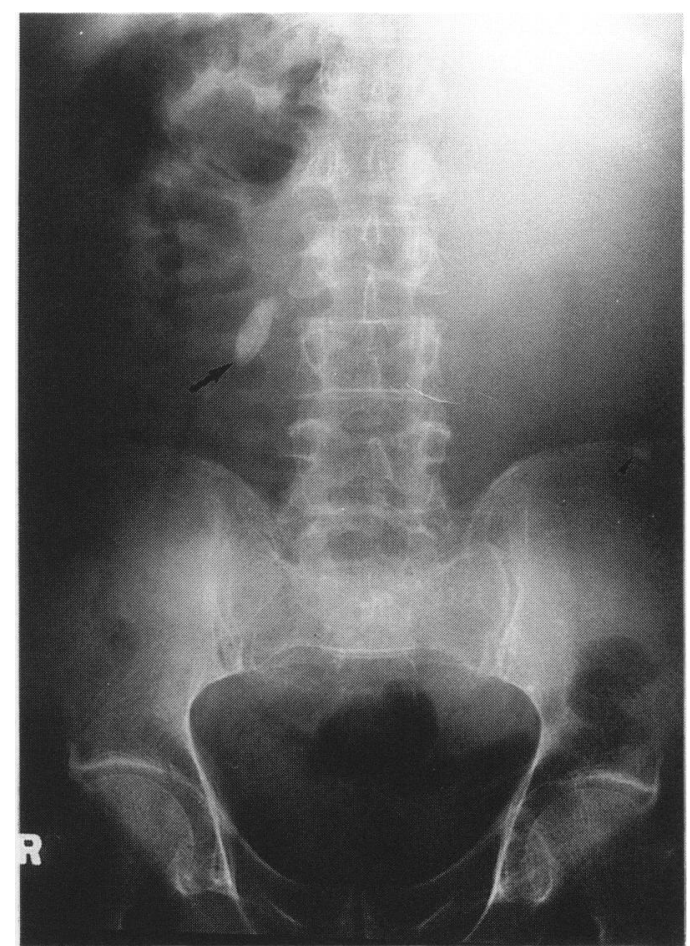

A

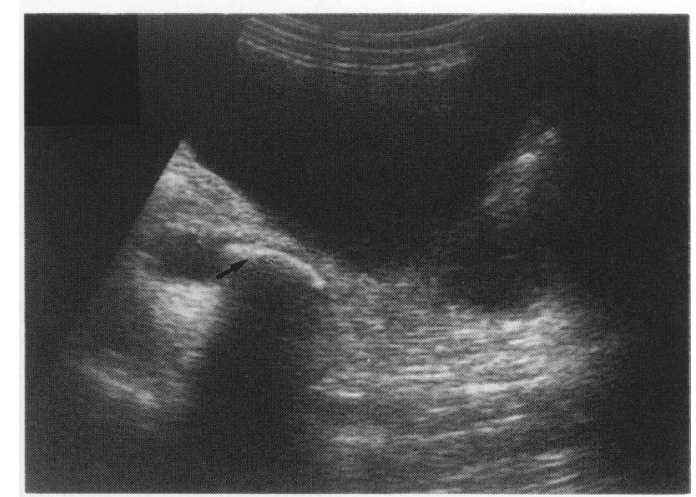

B

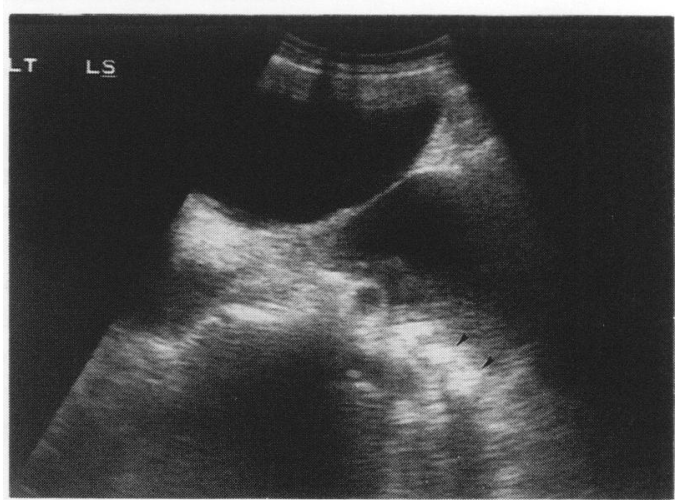

C

Figure 3 (A) Plain abdominal radiograph shows a large soft tissue opacity occupying the left hemiabdomen. An ovoid radio-opacity is seen overlying the right L4 transverse process (arrow) with multiple rounded radio-opacities in the left lower quadrant (arrowheads). (B,C) Longitudinal ultrasound scans of the left kidney demonstate left hydronephrosis with the obstructing left PUJ stone (arrow), and multiple calculi in the left lower pole calyx (arrowheads)

may lead to paracentesis with the possible consequence of pyelonephrosis, urinoma, sepsis or shock. The differential diagnosis 


\begin{tabular}{|l|}
\hline $\begin{array}{l}\text { Giant hydronephrosis: differential } \\
\text { diagnosis }\end{array}$ \\
\hline - ascites \\
- intraperitoneal cysts (mesenteric or \\
choledochal) \\
- retroperitoneal cysts (renal or adrenal or \\
pancreatic pseudocysts) \\
ovarian cysts/tumours \\
\hline
\end{tabular}

clinically includes intraperitoneal, and retroperitoneal cysts, pancreatic pseudocysts, and ovarian cysts/tumours (see box). Giant hydronephrosis tends to be a flabby tumour, while cysts generally maintain tension clinically.

The most important aspect of management is early diagnosis with accurate pre-operative delineation of anatomy of the affected kidney. Historically, the correct pre-operative diagnosis has been made in less than $50 \%$ of cases. ${ }^{11}$ Tombari et $a l^{11}$ reviewed 61 cases of surgically proven giant hydronephrosis and showed that the initial diagnosis was erroneous in $54 \%$. The initial clinical diagnosis in our three patients was also inaccurate. Ultrasound promptly confirmed the diagnosis in all patients whilst the calculi were not readily apparent on plain radiographs in cases 1 and 2 . Awareness of this condition by the clinician and radiologist would enable prompt diagnosis and appropriate therapy.

Radiologically, there is displacement of abdominal viscera to one side on the plain abdominal film or upper gastrointestinal series. Although the radiological hallmark has been a large radiodense homogenous mass in the region of the kidney that fails to register contrast medium on an excretory urogram, we find ultrasound to be a quick and sensitive method of establishing the diagnosis. The classical ultrasound features are a multiseptated cystic lesion with the 'cysts' communicating with one another. The diagnosis can be confidently made when communication between the dilated calyces and pelvis is demonstrated, and the cause of obstruction, often an ureteric calculus, is shown on ultrasound, as in our patients. The potential pitfall during ultrasound is misdiagnosis as a multicystic dysplastic kidney, which is distinctly uncommon in adults, and more often diagnosed in the paediatric population. The cystic spaces in this condition do not communicate with one another, producing the 'cluster of grapes' sign, and there is often contralateral renal abnormality. ${ }^{12}$ Other less common misdiagnoses at imaging include retroperitoneal and ovarian

1 Crooks KK, Hendren WH, Pfister RC. Giant hydronephrosis in children. $f$ Pediatr Surg 1979; 14: 844-50.

2 Friedland GW, Filly R, Goris M, et al. (eds) Congenital anomalies of the urinary tract. In: Uroradiology: an integrated approach. Vol 2. Edinburgh: Churchill Livingstone, grated approach. Vol
1983 , pp 1386-91.

3 Ochsner MG, Fuselier HA, Brannan W, et al. Congenital Ochsner MG, Fuselier HA, Brannan W, et al. Congen
giant hydronephrosis in adults. Urology 1977; 10: 422.

4 Macksood MJ, James RE. Giant hydronephrosis in ectopic Macksood MJ, James RE. Giant hydronephro
kidney in a child. Urology 1983; 22: 532-5.

5 Uson AC, Levitt SB, Lattimer JK. Giant hydronephrosis in Uson AC, Levitt SB, Lattimer JK. Giant
children. Paediatrics 1969; 44: 209-16.

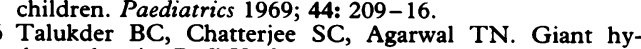
dronephrosis. Br $\mathcal{F}$ Urol 1979; 51: 322-3.

7 Livine PM, Huben RP, Pontes JE. Transitional cell carcinoma in a solitary giant hydronephrotic kidney. $\mathrm{Br} \mathcal{F}$ Urol 1986; 58: $727-8$.
Giant hydronephrosis: features

Clinical

- non specific.

- huge abdominal mass or abdominal distension, usually of long standing.

- flank pain and/or haematuria after mild trauma.

Radiological

- displacement of abdominal viscera to one side on plain abdominal film, may visualise radio-opaque urinary calculi as cause of obstruction.

- large dense homogenous mass in the region of the kidney that fails to register contrast medium on an excretory urogram

- ultrasound shows a large multi-septated cystic lesion with communication demonstrated between dilated calyces and pelvis.

Giant hydronephrosis: summary points

- definition: a kidney containing more than 11 of fluid in its collecting system.

- radiological criteria: hydronephrotic renal pelvis that meets or crosses the midline, occupies a hemi-abdomen, and extends for a length of five vertebrae or more.

- in adults, often misdiagnosed clinically when secondary to calculous obstruction

- plain radiographs may not provide an accurate diagnosis when there is obscuration of radio-opaque urinary calculi by bowel shadows or overlap with the spine.

- ultrasound is a quick and sensitive method of establishing the diagnosis.

tumours, and dilated fluid-filled bowel loops.

Once the suspicion of giant hydronephrosis secondary to calculous obstruction has been raised on ultrasound, isotope (DMSA) scanning is essential in the determination of differential renal function. Management of giant hydronephrosis is surgical. Nephrectomy is the usual method of treatment, as there is often very poorly functioning renal tissue. Nephrectomy rates, however, vary from $30-70 \%{ }^{1,3}$ A two-stage procedure with gradual pre-operative decompression is advocated to prevent possible cardiopulmonary collapse and alimentary tract dysfunction which might be caused by sudden decrease in intra-abdominal pressure. A more conservative approach involving reconstructive surgery (pyeloplasty) is generally reserved for the younger patient, patients with bilateral disease, or for kidneys with salvageable residual function.

8 Al Saleh BMS, Hadi AQ, Abdin MW. Giant hydronephrosis. Br f Urol 1992; 70: 686-7.

9 Morris SB, Dick JA. Ureteric obstruction secondary to contralateral hydronephrosis. Br $¥$ Radiol 1994; 67: 100-1. Krane RJ, Retik AB. Neonatal perirenal extravasation. $\dot{f}$ Urol 1974; 111: 96 .

11 Tombari AA, Power RF, Harper JM, et al. Giant hydronephrosis - a case report with review of the literature. $\mathcal{J}$ Urol 1968; 100: $120-3$.

12 Sanders RC, Hartman DS. The sonographic distinction between neonatal multicystic kidney and hydronephrosis. Radiology 1984; 151: 621 . 